\title{
Nonperturbative Debye Mass in Finite Temperature QCD
}

\author{
K. Kajantie, ${ }^{1,2}$ M. Laine,${ }^{3}$ J. Peisa, ${ }^{4}$ A. Rajantie,${ }^{2}$ K. Rummukainen, ${ }^{5}$ and M. Shaposhnikov ${ }^{1}$ \\ ${ }^{1}$ Theory Division, CERN, CH-1211 Geneva 23, Switzerland \\ ${ }^{2}$ Department of Physics, University of Helsinki, P.O. Box 9, 00014 Helsinki, Finland \\ ${ }^{3}$ Institut für Theoretische Physik, Philosophenweg 16, D-69120 Heidelberg, Germany \\ ${ }^{4}$ Department of Mathematical Sciences, University of Liverpool, Liverpool L69 3BX, United Kingdom \\ ${ }^{5}$ Fakultät für Physik, Postfach 100131, D-33501 Bielefeld, Germany
}

(Received 1 August 1997)

\begin{abstract}
Employing a nonperturbative gauge invariant definition of the Debye screening mass $m_{D}$ in the effective field theory approach to finite temperature QCD, we use 3D lattice simulations to determine the leading $\mathcal{O}\left(g^{2}\right)$ and to estimate the next-to-leading $\mathcal{O}\left(g^{3}\right)$ corrections to $m_{D}$ in the high temperature region. The $\mathcal{O}\left(g^{2}\right)$ correction is large and modifies qualitatively the standard power-counting hierarchy picture of correlation lengths in high temperature QCD. [S0031-9007(97)04353-6]
\end{abstract}

PACS numbers: 11.10.Wx, 11.15.Ha, 12.38.Mh

QCD matter, a spatially and temporally extended system of matter described by the laws of quantum chromodynamics, goes at high temperatures into a quark-gluon plasma phase, in which color is no more confined and chiral symmetry is restored. An essential quantity, describing coherent static interactions in the plasma, is the inverse screening length of color electric fields, the Debye mass $m_{D}$. The Debye mass enters in many essential characteristics of static properties of the plasma. Its numerical value is important for phenomenological discussions of formation of the quark-gluon plasma, for the analysis of $J / \Psi$ and $Y$ suppression in heavy ion collisions, for the computation of parton equilibration rates, etc. (see, e.g., [1]).

The definition and computation of the Debye mass for Abelian QED plasma is well understood [2]. The electromagnetic current $j_{\mu}$ is a gauge-invariant quantity, and the Debye mass can be extracted from the two-point gauge invariant correlation function of $j_{0}$ in the plasma. There are no massless charged particles in QED, which allows an infrared-safe perturbative computation of the Debye mass in powers of the electromagnetic coupling $e$. This has been done to order $e^{5}$ [3]. The situation in QCD is much more complicated. First, the corresponding current in QCD, $j_{\mu}^{a}$, is not a gauge-invariant quantity. Second, there are massless charged gluons which give rise to infrared divergences and prevent the perturbative determination of the Debye mass beyond leading order.

A nonperturbative gauge-invariant definition of the Debye mass in vectorlike theories with zero chemical potential was suggested in [4]. According to it, $m_{D}$ can be defined from the large distance exponential falloff of correlators of gauge-invariant time-reflection odd operators $O$,

$$
\langle O(\tau, \vec{x}) O(\tau, 0)\rangle \sim C|\vec{x}|^{\beta} \exp \left(-m_{D}|\vec{x}|\right),
$$

where $C$ and $\beta$ are some constants. The simplest choice for the operator $O$ is $F_{03}^{a} F_{12}^{a}$, and other examples can be found in [4]. In principle, four-dimensional lattice simulations of hot QCD would thus allow a measurement of the Debye mass at any temperature.
The aim of this Letter is a nonperturbative determination of the high temperature limit of the Debye mass, at $T>$ a few $\times T_{c}$. We will see that the effective 3D approach to high temperature gauge theories, developed in [5-7] (for a review, see [8]) allows a simple and transparent gauge-invariant definition of the Debye mass [4], while 3D lattice Monte Carlo simulations provide an economical way to determine its value. The corrections to the leading result we shall find are numerically large; thus many computations in the phenomenology of quark-gluon plasma in heavy ion collisions should be reanalyzed.

The theory we shall study is QCD with $N_{f}$ massless quark flavors and with the gauge group $\mathrm{SU}(N)$ with $N=$ 2,3 . At high temperatures and zero chemical potential the Debye mass can be expanded in a power series in the QCD coupling constant $g=g(\mu)$ [the scale $\mu$ will be specified later; the result for $N_{f}=0$ is shown explicitly in Eq. (5)]:

$$
\begin{aligned}
m_{D}= & m_{D}^{\mathrm{LO}}+\frac{N g^{2} T}{4 \pi} \ln \frac{m_{D}^{\mathrm{LO}}}{g^{2} T} \\
& +c_{N} g^{2} T+d_{N, N_{f}} g^{3} T+\mathcal{O}\left(g^{4} T\right) .
\end{aligned}
$$

The leading order (LO) perturbative result, $m_{D}^{\mathrm{LO}}=$ $\left(N / 3+N_{f} / 6\right)^{1 / 2} g T$, has been known for a long time [9]. The logarithmic part of the $\mathcal{O}\left(g^{2}\right)$ correction can be extracted perturbatively [10], but $c_{N}$ and the higher order corrections are nonperturbative. We are going to evaluate numerically the coefficients $c_{N}$ and $d_{N, N_{f}}$.

Static Green's functions for bosonic fields of high temperature QCD at distances $|x| \gg T^{-1}$ we are interested in can be determined by constructing an effective 3D gauge theory, containing static magnetic gluons and the zero component of the 4D gauge field, $A_{0}$ [5-7]. Moreover, a superrenormalizable 3D theory, defined by the Lagrangian

$$
\begin{aligned}
L_{\mathrm{eff}}\left[A_{i}^{a}, A_{0}^{a}\right]= & \frac{1}{4} F_{i j}^{a} F_{i j}^{a}+\operatorname{Tr}\left[D_{i}, A_{0}\right]\left[D_{i}, A_{0}\right] \\
& +m_{3}^{2} \operatorname{Tr} A_{0}^{2}+\lambda_{A}\left(\operatorname{Tr} A_{0}^{2}\right)^{2},
\end{aligned}
$$


gives the Green's functions to a relative accuracy $\mathcal{O}\left(g^{4}\right)$ [6], which is sufficient for the accuracy of the expansion in Eq. (2). The parameters of the effective theory are related to the parameters of $4 \mathrm{D} \mathrm{QCD}\left(\Lambda_{\overline{\mathrm{MS}}}, N, N_{f}\right)$ and the temperature as described in [11]. For brevity, we give here the explicit expressions only for $N_{f}=0$ :

$$
\begin{gathered}
g_{3}^{2}=g^{2}\left(4 \pi e^{-\gamma_{E}-\frac{1}{22}} T\right) T=\frac{24 \pi^{2} T}{11 N \ln \left(6.742 T / \Lambda_{\overline{\mathrm{MS}}}\right)}, \\
m_{3}^{2}=\frac{N}{3} g^{2}\left(4 \pi e^{-\gamma_{E}-\frac{5}{22}} T\right) T^{2}, \\
\lambda_{A}=\frac{6+N}{24 \pi^{2}} g^{4}\left(4 \pi e^{-\gamma_{E}-\frac{7}{44}} T\right) T .
\end{gathered}
$$

Here $g(\mu)$ is the QCD coupling in the $\overline{\mathrm{MS}}$ scheme and all the effective theory couplings have been computed including both the leading and the next-to-leading order contributions. The couplings (4)-(6) are independent of the gauge chosen for the perturbative computation. The expansion parameter is $\sim \alpha_{s} / \pi$ so that the result should be accurate down to $T \approx$ a few $\times \Lambda_{\overline{\mathrm{MS}}}$.

The dynamics of the 3D effective theory is fully characterized by the two dimensionless ratios

$$
y=\frac{m_{3}^{2}}{g_{3}^{4}}, \quad x=\frac{\lambda_{A}}{g_{3}^{2}},
$$

and by the dimensionful coupling $g_{3}^{2}$. The value of $x$ is essentially fixed by $T$,

$$
\begin{aligned}
x & =\frac{6+N}{24 \pi^{2}} g^{2}\left(4 \pi e^{-\gamma_{E}-3 / 11} T\right) \\
& =\frac{6+N}{11 N} \frac{1}{\ln \left(5.371 T / \Lambda_{\overline{\mathrm{MS}}}\right)},
\end{aligned}
$$

while $y$ and $x$, corresponding to physical 4D finite $T$ QCD for $N_{f}=0$, are related by

$$
\begin{aligned}
y=y_{\mathrm{dr}}(x) & =\frac{2}{9 \pi^{2} x}+\frac{1}{4 \pi^{2}}+\mathcal{O}(x), \quad N=2 \\
& =\frac{3}{8 \pi^{2} x}+\frac{9}{16 \pi^{2}}+\mathcal{O}(x), \quad N=3 .
\end{aligned}
$$

We are now ready to give a gauge-invariant definition of the Debye mass in the 3D language [4]. Physically, we want a local operator which makes $A_{0}^{a}$ gauge invariant in the 3D theory and contains $A_{0}^{a}$ singly. We can single out this state by a symmetry consideration. Note that the effective Lagrangian (3) has a discrete symmetry $A_{0} \leftrightarrow$ $-A_{0}$. Then the Debye mass can be defined as the mass of the lightest 3D state which is odd under this symmetry. It is reasonable to expect that the lightest state couples to the lowest dimensional operator which contains only one insertion of $A_{0}$ :

$$
h_{i}=\epsilon_{i j k} \operatorname{Tr} A_{0} F_{j k} .
$$

Here the field $A_{0}^{a}$ has been made gauge invariant by dressing it with a cloud of magnetic gluons. The use of different "blocking levels" [11] extends the basis of the operators considered, but in principle one should also consider other operators such as $\operatorname{Tr} A_{0}^{3}$ (for $N \geq 3$ ). We regard it as unlikely, though, that the inclusion of this operator could modify the picture. First, the heavy quark expansion (see below) suggests that the parametrically dominant mass term for this operator is $\sim 3 m_{D}^{\mathrm{LO}}$. Second, it would be somewhat odd if the $N=2$ case, where this operator is absent, were qualitatively different from the $N=3$ case. Third, the final result (15) shows a natural $N$ dependence which can hardly be a coincidence.

At high $T$, one has $g \ll 1$ and, according to Eqs. (4) and (5), $m_{3} \gg g_{3}^{2}$. This is the "heavy quark limit" of the 3D theory, in which the mass $m_{D}$ of the singlet state is dominated by the bare mass $m_{3}$ of the scalar "quark" $A_{0}^{a}$. For dimensional reasons, the exact mass $m_{D}$ can in this limit be expanded as

$$
m_{D}=m_{3}+a_{N} g_{3}^{2}+\frac{b_{N} g_{3}^{4}}{m_{3}}+\mathcal{O}\left(\lambda_{A}, \frac{g_{3}^{2} \lambda_{A}}{m_{3}}, \frac{g_{3}^{6}}{m_{3}^{2}}, \ldots\right),
$$

where $a_{N}$ and $b_{N}$ are constants, perhaps involving a logarithm of $m_{3} / g_{3}^{2}$. The terms neglected are of higher order using the power counting in Eqs. (4)-(6). Comparing Eqs. (2) and (12), one sees that

$$
\begin{aligned}
a_{N} & =\frac{N}{4 \pi} \ln \frac{\sqrt{N / 3+N_{f} / 6}}{g}+c_{N}, \\
d_{N, N_{f}} & =\frac{b_{N}}{\sqrt{N / 3+N_{f} / 6}} .
\end{aligned}
$$

Here we used the fact that the scale dependence of the nonperturbative terms in Eq. (2) is at least of order $\mathcal{O}\left(g^{4}\right)$. Since the expansion (12) refers only to the 3D theory, the constants $a_{N}$ and $b_{N}$ depend on $N$ but clearly not on $N_{f}$. Thus $c_{N}$ is $N_{f}$ independent, while $d_{N, N_{f}}$ depends on $N_{f}$ only through $m_{D}^{\mathrm{LO}}$.

In terms of our dimensionless variables (7), Eq. (12) becomes

$$
\frac{m_{D}}{g_{3}^{2}}=\sqrt{y}+\frac{N}{4 \pi} \ln \sqrt{y}+c_{N}+\frac{b_{N}}{\sqrt{y}}+\ldots
$$

The mass $m_{D}$ can now be measured by putting the effective 3D theory on the lattice [11] and by measuring the exponential falloff of the correlator $\left\langle h_{3}\left(x_{3}\right) h_{3}(0)\right\rangle \sim$ $\exp \left(-m_{D}\left|x_{3}\right|\right)$, where $h_{3}\left(x_{3}\right)$ is summed over the transverse $\left(x_{1}, x_{2}\right)$ plane [12]. To make simulations for fixed continuum parameters $x, y$, one has to employ the latticecontinuum relations derived in [13]. The correlation function is measured both with zero and finite transverse momentum, and in order to enhance the overlap with the asymptotic state the measurements are performed with several levels of recursive blocking of the operators. We select the blocking level and momentum sector which has the best signal for the asymptotic mass separately for each 
Monte Carlo run. Since the longest correlation length in each case is less than $1 / 5$ of the linear size of the lattice, we expect the finite volume effects to be negligible in comparison with the statistical errors. This was also explicitly checked by performing simulations with different volumes in isolated cases.

The mass $m_{D}$ is defined in the whole $y, x$ parameter space. To have results which are relevant for $4 \mathrm{D}$ physics, we perform the measurements along the two-loop dimensional reduction lines $y_{\mathrm{dr}}(x)$, Eqs. (9) and (10). To measure the coefficients in Eq. (14) one should use the part of this curve corresponding to $\sqrt{y} \gg 1$. The results for $N=3$ are shown in Fig. 1, in units of 4D $g T$ $\left(=g_{3}^{2} \sqrt{3 y / N}\right.$ in 3D units). The Monte Carlo runs are performed with several lattice spacings $a$, parametrized by $\beta_{G} \equiv 2 N /\left(g_{3}^{2} a\right)$. For $\mathrm{SU}(3) \beta_{G}$ varies by more than an order of magnitude (although not at the same value of $x$ ), as shown in Fig. 1; for $\mathrm{SU}(2)$, the measurements are done with $\beta_{G}=20$ and 32. The top scale of Fig. 1 shows the physical temperature $T / \Lambda_{\overline{\mathrm{MS}}}$ along the $y_{\mathrm{dr}}(x)$ line. Note that the highest temperatures are larger than $10^{100} \times \Lambda_{\overline{\mathrm{MS}}} \sim 10^{100} \times T_{c}$.

At small $x$ (large $y$ ), the fit to the function (12) is very good, as indicated by the continuous line in Fig. 1. In order to see in detail the sensitivity of the fit to the parameters, in Fig. 2 we replot the SU(3) data (restricted to $x<0.05)$ in terms of the quantity $\delta m / g_{3}^{2}=m_{D} / g_{3}^{2}-$ $\sqrt{y}-\frac{N}{4 \pi} \ln \sqrt{y}$ as a function of $1 / \sqrt{y}$. The intersection of the curve with the vertical axis gives the value of $c_{N}$

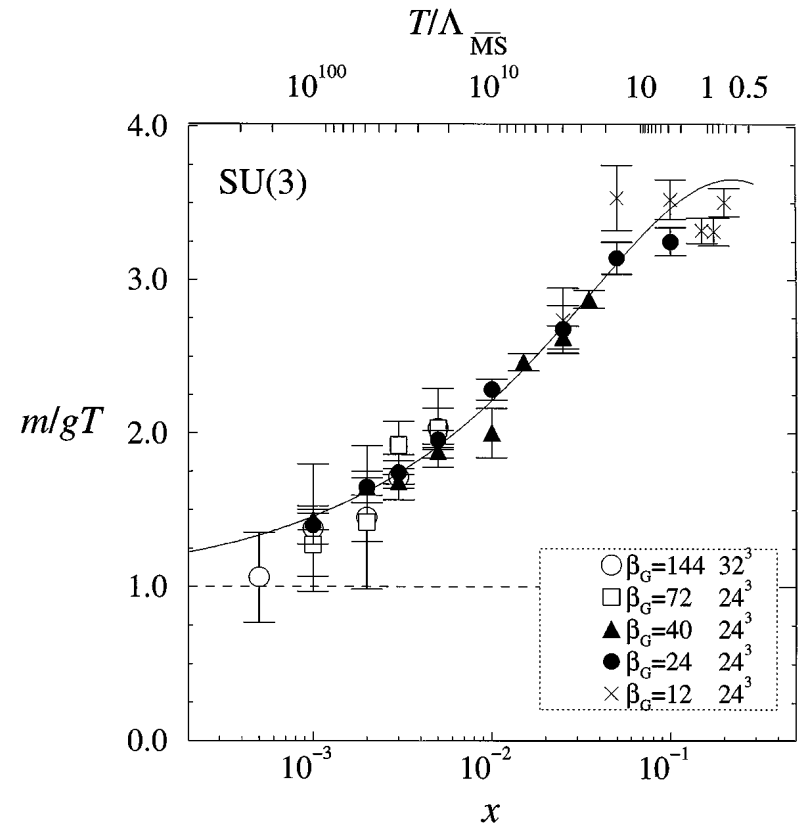

FIG. 1. The gauge invariant Debye mass for $\mathrm{SU}(3)$, as a function of $x$, or $T / \Lambda \overline{\mathrm{MS}}$ through Eq. (8). The scale of $g$ on the $y$ axis is fixed according to Eq. (5). The dashed line marks the leading order $m_{D}^{\mathrm{LO}}$, and the continuous line the two-parameter fit to Eq. (12) with the parameters as in Eq. (15). and the slope gives $b_{N}=d_{N, N_{f}} \sqrt{N / 3+N_{f} / 6}$. One can see that the linear fit is rather good even down to small values of $\sqrt{y}$. The large nonzero value of the intercept is very robustly determined. The slope $b_{N}$ is small and has a relatively large error. Only the statistical error is given, but the value of $b_{N}$ also depends on the range of $y^{-1 / 2}$ included.

The results of the fits are

$$
\begin{array}{ll}
\mathrm{SU}(2): c_{N}=1.58 \pm 0.20, & b_{N}=-0.03 \pm 0.25, \\
\mathrm{SU}(3): c_{N}=2.46 \pm 0.15, & b_{N}=-0.49 \pm 0.15 .
\end{array}
$$

The large number $c_{N}$ is related to nonperturbative 3D effects, while the smaller $d_{N, N_{f}}$ can be viewed as being related to the choice of scale in $m_{D}^{\mathrm{LO}}$. For $N=2$ we can in practice verify only that $d_{2, N_{f}}$ is close to zero. Note that writing $c_{N}=N \tilde{c}_{N}$, one has $\tilde{c}_{N}=0.79 \pm 0.10(N=2)$, $0.82 \pm 0.05(N=3)$.

One can observe the following:

(i) The leading term is dominant only at extremely large $T$. For $\mathrm{SU}(3)$, the leading term is larger than the $\mathcal{O}\left(g^{2}\right)$ correction for $g<1 / 2.46$ or for $T / \Lambda_{\overline{\mathrm{MS}}} \gtrsim$ $\exp \left(8 \pi^{2} 2.46^{2} / 11\right) \gtrsim 10^{19}$. This implies that the leading term dominates only when QCD merges into a unified theory anyway.

(ii) The four terms in Eq. (14) fit the data over all the range $T \geqslant 100 T_{c}$ rather well, and there is no need for further corrections.

(iii) In the range $\Lambda_{\overline{\mathrm{MS}}} \approx T_{c} \lesssim T \lesssim 100 T_{c}, m_{D}$ is rather constant and $\approx 3.0 \mathrm{~m}_{D}^{\mathrm{LO}}$ for $\mathrm{SU}(2)$ and $\approx 3.3 \mathrm{~m}_{D}^{\mathrm{LO}}$ for SU(3). It should be noted, though, that in this regime $m_{D}^{\mathrm{LO}}>T$ so that the hierarchy $m_{D}^{2} /(2 \pi T)^{2} \ll 1$ required

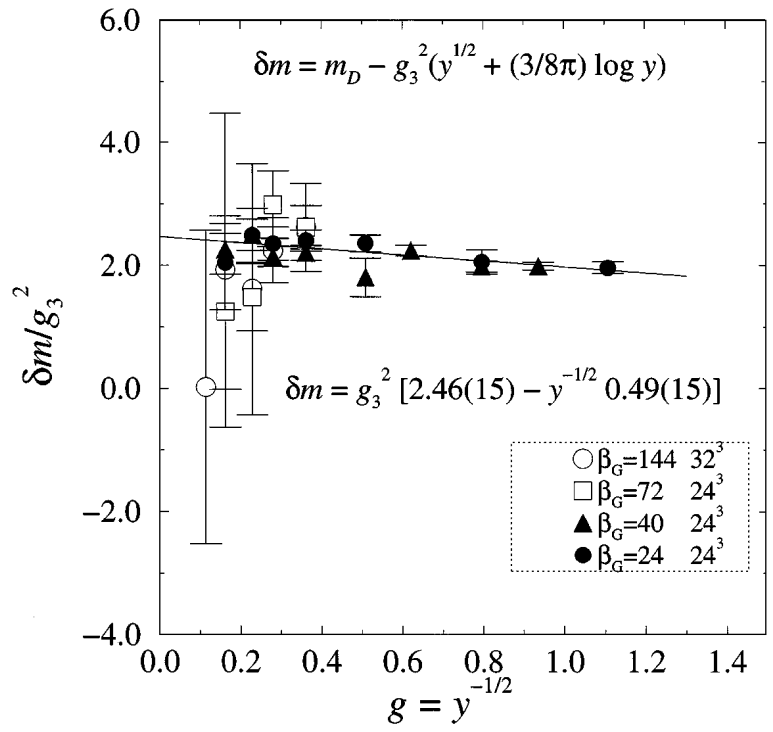

FIG. 2. The corrections of order $\mathcal{O}\left(g_{3}^{2}\right)$ and $\mathcal{O}\left(g_{3}^{2} / \sqrt{y}\right)$ to the $\mathrm{SU}(3)$ screening mass $m_{D}$ in $3 \mathrm{D}$ units, corresponding to Eq. (14). 
for an accurate description of $4 \mathrm{D}$ physics through a 3D effective theory is getting weaker.

(iv) The mass measured from the $\left\langle A_{0} A_{0}\right\rangle$ correlator in the Landau gauge in $4 \mathrm{D}$ simulations for $N_{f}=0$ has also been observed to be clearly larger than the leading term [14].

(v) If the mass $\sim g T$ of the $A_{0}^{a}$ field is "large," larger than the nonperturbative $\mathcal{O}\left(g^{2}\right) T$ correction, the $A_{0}^{a}$ field can be further perturbatively integrated out and a simpler effective theory, containing only $A_{i}^{a}$ (and possible scalar fields) can be derived [5-7]. Our results imply that this can be accurately carried out for QCD only at extremely high temperatures, $T \gg T_{c}$. In the electroweak case the accuracy of the integration is sufficient even for $T \sim$ $T_{c}$ both since the leading term has a bigger coefficient $\left(m_{D}^{\mathrm{LO}}=\sqrt{11 / 6} g T\right)$ than the $N_{f}=0$ QCD considered here and since in the relevant $T$ regime the coupling constant $g=g\left(m_{W}\right) \approx 2 / 3$ is smaller.

(vi) The usual parametric "power counting" picture of correlation lengths in high temperature QCD says that the longest scale, related to the magnetic sector of the theory, is $m_{M}^{-1} \sim\left(\text { const } \times g^{2} T\right)^{-1}$. A shorter scale, $\sim(g T)^{-1}$, is associated with Debye screening. Our results show that this picture can be quantitatively correct only at extremely large temperatures. Indeed, purely magnetic effects, as measured by the 3D glueball (operator $F_{i j}^{a} F_{i j}^{a}$ ) mass $\left(m_{G} \approx 2 g_{3}^{2}\right.$ for pure $\left.\mathrm{SU}(2)[11,15]\right)$ tend to be numerically large, so that $m_{M} \sim m_{D} \gtrsim(2-3) T$ in a very wide range of temperatures, at least up to $T \sim 10^{3} T_{c}$ (This gauge invariant result is in contrast to the small magnetic gluon masses measured in Landau gauge [14].) In this range the longest length scale corresponds to a scalar $0^{++} 3 \mathrm{D}$ "bound state" of two $A_{0}$ quanta, associated with the operator $A_{0}^{a} A_{0}^{a}$ (the power counting suggestion that this state is roughly twice as heavy as $m_{D}$ holds again only at extremely high $T$ ).

Summarizing, we have carried out with lattice Monte Carlo techniques a gauge independent measurement of the Debye mass in finite $T$ QCD. The measurement is based on first deriving with two-loop perturbative computations a 3D effective theory. The expansion parameter is $\sim \alpha_{s} / \pi$, so that the result is accurate down to $T$ close to $T_{c}$. The mass is obtained by measuring correlators of the gauge-invariant local operator $A_{0}^{a} F_{j k}^{a}$ in the $3 \mathrm{D}$ theory. The leading and next-to-leading corrections to $m_{D}$ were determined and found to be large. In fact, for temperatures from $T_{c}$ up to $T \sim 1000 \Lambda \overline{\mathrm{MS}}$ the nonperturbative
Debye screening mass is about a factor of 3 larger than the lowest order estimate.

It remains to be seen whether this modification of the standard picture of high-temperature gauge theories has applications in the cosmological discussion of the quarkhadron phase transition or in the phenomenology of heavy ion collisions.

The simulations were carried out with a Cray C94 and Cray T3E at the Finnish Center for Scientific Computing.

[1] F. Karsch, M. Mehr, and H. Satz, Z. Phys. C 37, 617 (1988); X.-N. Wang, Phys. Rep. 280, 287 (1997).

[2] I. Akhiezer and S. Peletminski, Zh. Eksp. Teor. Fiz. 38, 1829 (1960) [Sov. Phys. JETP 11, 1316 (1960)].

[3] J.-P. Blaizot, E. Iancu, and R. Parwani, Phys. Rev. D 52, 2543 (1995).

[4] P. Arnold and L. G. Yaffe, Phys. Rev. D 52, 7208 (1995).

[5] P. Ginsparg, Nucl. Phys. B170, 388 (1980); T. Appelquist and R. Pisarski, Phys. Rev. D 23, 2305 (1981); S. Nadkarni, Phys. Rev. D 27, 917 (1983).

[6] K. Kajantie, K. Rummukainen, and M. Shaposhnikov, Nucl. Phys. B407, 356 (1993); K. Kajantie, M. Laine, K. Rummukainen, and M. Shaposhnikov, Nucl. Phys. B458, 90 (1996).

[7] E. Braaten and A. Nieto, Phys. Rev. D 51, 6990 (1995); Phys. Rev. D 53, 3421 (1996).

[8] M.E. Shaposhnikov, Proceedings of the International School of Subnuclear Physics on Effective Theories and Fundamental Interactions, Erice, 1996 (World Scientific, River Edge, NJ, 1997).

[9] E. Shuryak, Zh. Eksp. Teor. Fiz. 74, 408 (1978) [Sov. Phys. JETP 47, 212 (1978)]; J. Kapusta, Nucl. Phys. B148, 461 (1979); D. Gross, R. Pisarski, and L. Yaffe, Rev. Mod. Phys. 53, 43 (1981).

[10] A. K. Rebhan, Phys. Rev. D 48, R3967 (1993); Nucl. Phys. B430, 319 (1994).

[11] K. Kajantie, M. Laine, K. Rummukainen, and M. Shaposhnikov, Nucl. Phys. B (to be published), hep-ph/ 9704416.

[12] The coefficient $c_{N}$ could also be measured from the perimeter law behavior of a large adjoint Wilson loop in the pure 3D $\operatorname{SU}(N)$ gauge theory [4].

[13] M. Laine and A. Rajantie, Heidelberg University Report No. HD-THEP-97-16, hep-lat/9705003.

[14] U. Heller, F. Karsch, and J. Rank, in Proceedings of Lattice '97 [Nucl. Phys. B (Proc. Suppl.) (to be published)]; hep-lat/9708009.

[15] O. Philipsen, M. Teper, and H. Wittig, Nucl. Phys. B469, 445 (1996). 